\title{
Design and Development of Low Cost, High Precision Continuous Positive Airway Pressure (CPAP) Machine
}

\author{
${ }^{* 1}$ Rohan Bang, ${ }^{2}$ Shubham Mundra, ${ }^{3}$ Indu Mandwi \\ ${ }^{1,2}$ CSE Department GHRCE, Nagpur, ${ }^{3}$ Assistant Professor GHRCE, Nagpur \\ Email:*rohanbang123@gmail.com, shubhamdmundra@gmail.com,indu.mandwi@ raisoni.net.com
}

Received: 09 ${ }^{\text {th }}$ July 2018, Accepted: $14^{\text {th }}$ August 2018, Published: $31^{\text {st }}$ August 2018

\begin{abstract}
Sleep Apnea (or Sleep Apnoea) means lack of breath during sleep; it is a condition where a person's breathing is interrupted for several seconds, multiple times at night, the oxygen supply in the blood and subsequently to the brain declines. It is a dangerous state which causes many people and is linked with many health related problems. It is because of airway collapsing or becoming blocked during sleep. This is a common disorder affecting about $30 \%$ of the population, moreover WHO has also reported sleep apnea is one of chronic respiratory disease. Sleep apnea is characterised in two types CSA (Central Sleep Apnea) and OSA (Obstructive Sleep Apnea). CSA, happens when the brain is unsuccessful to send the proper signals to the breathing muscles to start respiration, on the other hand OSA is caused by the complete or partial blockage in upper airway. By the use of Continuous Positive Airway Pressure (CPAP) machine a person who is having OSA can have a nice sleep as this machine increases air pressure in the throat so that airway does not get blocked. Existing machines in the market are costly. This paper presents a simple, low cost technique for the development of CPAP Machine.
\end{abstract}

Keywords: Sleep Apnea, Low Cost, High Precision, CPAP, Continuous Positive Airway Pressure Machine

\section{Introduction}

Sleep Apnea means lack of breath during sleep. It is because of airway collapsing or becoming blocked during sleep. Sleep apnea is characterized into two types: Central (CSA) and Obstructive (OSA).

A. Central Sleep Apnea

Central Sleep Apnea (CSA) eventuates when the brain is unsuccessful to send the proper signals to the breathing muscle to initiate respiration. Respiratory movements are either in the state of apnea (absent). Central sleep apnea can occur to anyone that is any age groups and any gender.

B. Obstructive Sleep Apnea

Obstructive Sleep Apnea (OSA) eventuates when the pharyngeal wall collapse due to relaxation of pharyngeal muscles during sleep leads to complete or partial blockage in upper airway. In many people, airway (windpipe) is obstructed because of a large or floppy tongue, excess tissue in the throat, obesity, nasal obstruction, or enlarged tonsils or uvula.

Diagnosis and available therapies

Diagnoses are based on signs and symptoms like loud snoring that can to disturb your sleep. It is recommended to have an overnight monitoring of your breath during sleep.

Numbers of therapies are available in the market like Continuous Positive Airway Pressure (CPAP), Oral appliance and various surgeries like uvulopalatopharyngoplasty, maxillomandibular, plastic rods implants and tracheostomy.

A. CPAP (Continuous positive airway pressure) Continuous positive airway pressure machine is used by a person who is having OSA for having a nice sleep, this machine increases air pressure in the throat such that compressed air acts as a lofty splint which prevent airway collapse or get blocked. Fig.4 is a prototype of CPAP machine which is been designed by us aimed for better performance, quick response and cost efficient model.

B. OAT (Oral Appliance Therapy)

Oral appliance therapy is designed to keep your throat open. It consists of a plastic (acrylic) oral gadget which fits much like a sports mouth guard. These appliances can alleviate OSA symptoms by improving blood oxygen saturation.

C. UPPP (Uvulopalatopharyngoplasty) Uvulopalatopharyngoplasty is a surgery in which tissue from the rear, top parts of the throat are removed, and in some conditions, tonsils and adenoids are removed.

D. MMA (Maxillomandibular)

Maxillomandibular is a surgery in which jaw is stepped ahead from your face bones making the space behind the tongue and behind the soft palate.

E. Implants

In implants, plastic rods are surgically implanted into the soft palate of the throat making obstruction less likely to happen.

F. Tracheostomy

Tracheostomy is a surgical procedure in which the opening is created in the fore most of the neck such that the tube can be shove into the windpipe (trachea) to help an individual breathe. 


\section{Proposed Methodology}

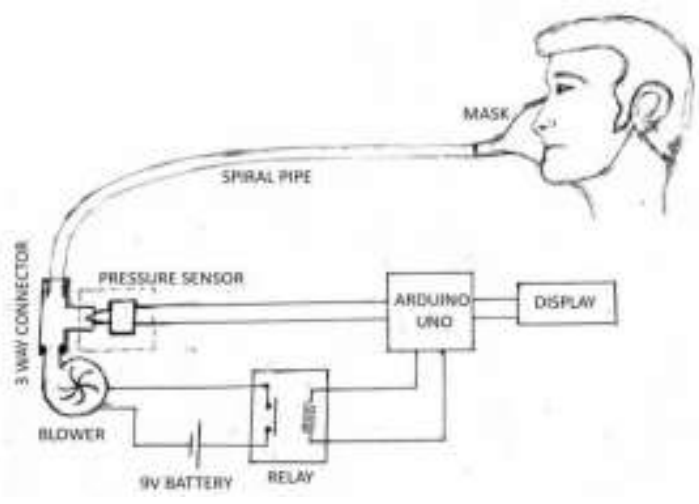

Fig.1. Schematic of the CPAP Major Components

Schematic diagram of the main components of the CPAP unit is as shown in Fig.1. Initially, air is passed to the blower. The blower is controlled by Arduino $\mathrm{UNO}$; pressure value at the nasal mask is taken from the pressure sensor for controlling the system.

\section{A. Materials and Methods}

CPAP Mask and Pipe: Selecting the perfect mask can be difficult but right sized CPAP mask is the integral need for obtaining effective CPAP therapy. Validating the detection of the leak is difficult for the patient as flow estimation is needed to know how much flow is for a quality sleep. Arduino UNO: An open source hardware and software company which design $\mu \mathrm{P}$ and $\mu \mathrm{C}$ kits Blower and Pressure sensor: Selecting the blower and pressure sensor was the most difficult part. The sensor needs to be very sensitive to very low pressure range.

\section{B. Software Part}

Designing the sketch of the machine was done in Proteus Design Suite which is a software tool suite used primarily for electronic design automation moreover the 3D Visualization Tool for an electronics designing. Mainly we have used Arduino which is an open source easy-to-use hardware and software. The language used is Embedded C.

\section{Control and System Design}

The flowchart of the algorithm of CPAP machine is as shown in Fig.2. The pressure value from the mask is taken and the required voltage of blower is been calculated. The design of the system is made such a way that the machine is cost efficient.

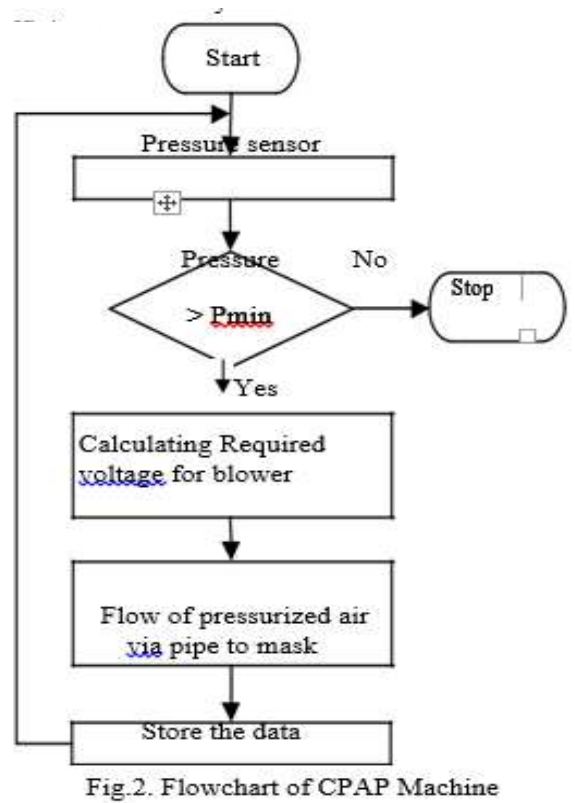

D. Hardware implementation

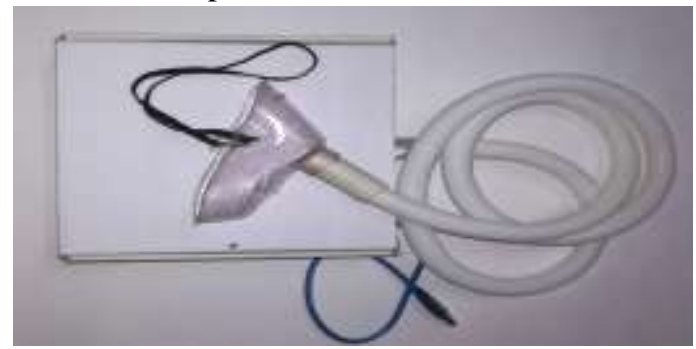

Fig.3. Assembly of Machine

The assembly of our CPAP machine is shown in Fig.3 and prototype of the machine designed by us, aimed better performance, quick response and cost efficient model is shown in Fig.4. There is a changeable DC pressure blower. The blower compresses purified atmospheric air which is controlled by Arduino UNO which is typical an 8-bit AVR, the pressure value of the mask is taken from the pressure sensor and data is send to Arduino UNO for controlling the system.

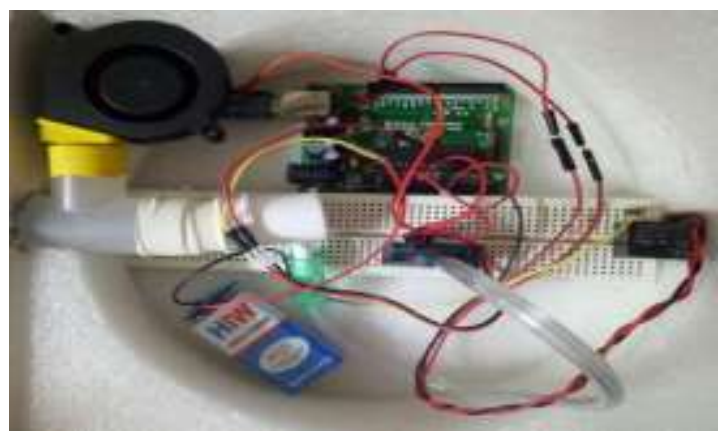

Fig.4. Prototype CPAP Machine 


\section{Results and Conclusion}

Sleep apnea is fast growing disorder spreading among all age groups. Previously people were not aware of this disorder but now we are having CPAP machine to make their life comfortable. Presently in the market, there are various brands making this machine whose cost is from 50 thousand to 2 lakh Indian rupee but we have made a similar prototype cost efficient CPAP machine.

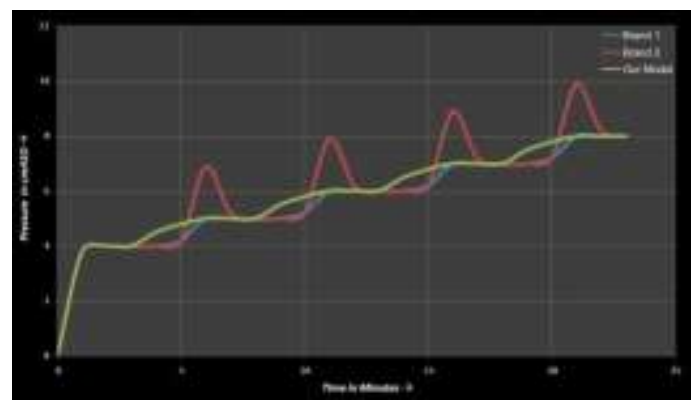

Fig 5 Graphical Analysis

We created a typical apnea state at $4^{\text {th }}$, 9 th, $14^{\text {th }}$ and $19^{\text {th }}$ minute the Fig.5 graph shows our machine gives better performance and quick response

\section{Acknowledgement}

We would like express our sincere gratitude toward our college GHRCE for continuous guidance and support during the project and special thanks to our grandmother Smt. Shridevi Bang, from where the idea of making this machine came.

\section{References}

[1] Aditya Sundar, Chinmay Das, "Low Cost, High Precision System for Diagnosis of Central Sleep Apnea Disorder", Department of Electronics, Electrical \& Instrumentation BITS Pilani, K.K. Birla Goa Campus Goa, India, International Conference on Industrial Instrumentation and Control (ICIC), College of Engineering Pune, India, May 28-30, 2015 [2] Joonas Paalasmaa "Monitoring Sleep with Force Sensor", PhD Thesis, Series of Publications A, Report A-2014-2 Helsinki, Measurement Department of Computer Science, University of Helsinki, Finland, January, 2014

[3] World Health Organization (WHO), "Global Surveillance, Prevention and Control of Chronic Respiratory Disease. A comprehensive approach,", pp. 32-34, 2007.

[4] Khosrow Behbehani, Fu-Chung Yen, John R. Burk, Edgar A. Lucas and John R. Axe "Automatic Control of Airway Pressure for Treatment of Obstructive Sleep Apnea", IEEE Transactions On Biomedical Engineering, Vol. 42, No. 10, October 1995
[5] Contemporary Reviews in Cardiovascular Medicine, Sleep Apnea and Cardiovascular Disease A Bidirectional Relationship Takatoshi Kasai, MD, $\mathrm{PhD}$; John S. Floras, MD, DPhil; T. Douglas Bradley, MD

[6] Central sleep apnea. Javaheri S1, Dempsey JA, Compr Physiol. 2013 Jan;3(1):141-63. doi: 10.1002/cphy.c110057.

[7] Aditya Sundar, Chinmay Das, "Low Cost, High Precision System for Diagnosis of Central Sleep Apnea Disorder", Department of Electronics, Electrical \& Instrumentation BITS Pilani, K.K. Birla Goa Campus Goa, India, International Conference on Industrial Instrumentation and Control (ICIC), College of Engineering Pune, India, May 28-30, 2015 [8] Kang, M., Mask Leak Investigation - CPAP Performance, in AR Investigational Devices, ResMed Ltd: Bella Vista, NSW- 2012

[9] Nicolas Grandjean-Thomsen, Martin Kang, and Gordon Malouf "Validation of Automatic CPAP Leak Algorithm", 35th Annual International Conference of the IEEE EMBS Osaka, Japan, 3 - 7 July, 2013 